\title{
Efficient callus formation and plant regeneration are heritable characters in sugar beet (Beta vulgaris L.)
}

\author{
Hiroyo Kagami ${ }^{1}$, Kazunori Taguchi ${ }^{2}$, Takumi Arakawa ${ }^{1}$, Yosuke Kuroda ${ }^{2}$, Hideto Tamagake ${ }^{3}$ and Tomohiko Kubo ${ }^{1 *}$ (D)
}

\begin{abstract}
Background: Obtaining dedifferentiated cells (callus) that can regenerate into whole plants is not always feasible for many plant species. Sugar beet is known to be recalcitrant for dedifferentiation and plant regeneration. These difficulties were major obstacles for obtaining transgenic sugar beets through an Agrobacterium-mediated transformation procedure. The sugar beet line 'NK-219mm-O' is an exceptional line that forms callus efficiently and is easy to regenerate, but the inheritance of these characters was unknown. Another concern was whether these characters could coexist with an annual habitat that makes it possible to breed short life-cycle sugar beet suitable for molecular genetic analysis.
\end{abstract}

Findings: Five sugar beet lines including NK-219mm-O were crossed with each other and subjected to in vitro culture to form callus. $F_{1} s$ with a NK-219mm-O background generally formed callus efficiently compared to the others, indicating that efficient callus formation is heritable. The regeneration potential was examined based on the phenotypes of calli after placement on regeneration medium. Five phenotypes were observed, of which two phenotypes regenerated shoots or somatic embryo-like structures. Vascular differentiation was evident in regenerable calli, whereas non-regenerable calli lacked normally developed vascular tissues. In a half-diallel cross, the callus-formation efficiency and the regeneration potential of reciprocal $F_{1} s$ progeny having a NK-219mm-O background were high. Finally, we crossed NK-219mm-O with an annual line that had a poor in vitro performance. The callus-formation efficiency and the regeneration potential of reciprocal $F_{1}$ were high. The regenerated plants showed an annual habitat.

Conclusions: Efficient callus formation and the high plant regeneration potential of NK-219mm-O were inherited and expressed in the $F_{1}$. The annual habitat does not impair these high in vitro performances.

Keywords: Dedifferentiation, DNA marker, $F_{1}$ hybrid, in vitro culture, Somatic embryo

\section{Findings}

Transgenic plants play pivotal roles in molecular genetic analysis and crop biotechnology. To obtain transgenic plants, Agrobacterium-mediated transformation techniques have been devised for many crops [1]. In some cases, explants are dedifferentiated in vitro to obtain callus that is subsequently infected with Agrobacterium harboring a recombinant Ti plasmid that will be inserted into plant chromosomes. The transformed cells are subsequently induced to regenerate whole plants.

\footnotetext{
* Correspondence: tomohiko@abs.agr.hokudai.ac.jp

${ }^{1}$ Research Faculty of Agriculture, Hokkaido University, Sapporo 060-8589, Japan

Full list of author information is available at the end of the article
}

Sugar beet (Beta vulgaris L.) is generally recalcitrant for dedifferentiation and plant regeneration, although cultivars and breeding lines show different responses to in vitro culture. This finding suggests that dedifferentiation and plant regeneration may be heritable characters and the genes involved in these characters may be scarce in sugar beet populations. In fact, Tomita et al. [2] investigated 61 sugar beet lines regarding their responses to in vitro culture and found variation in the frequencies of callus formation and somatic-embryo formation; however, the inheritance of these characters was not examined.

The sugar beet line 'NK-219mm-O', developed by the Hokkaido Agricultural Research Center (HARC), Japan, is an exceptional genotype that forms callus and regenerates 
plants very efficiently. These attributes enabled us to develop an Agrobacterium-mediated transformation system for sugar beet $[3,4]$. Using this system, molecular analyses of several genes have been completed [5-7]. On the other hand, the shortcomings of NK-219mm-O include its vernalization requirement, i.e. the line must experience a certain duration of low temperatures as a prerequisite for flowering. Examining, for example, flower phenotypes in transgenic sugar beet takes a long time due to the vernalization requirement, which is a rate limiting step for the molecular genetic analysis of sugar beets.

The vernalization requirement in sugar beet is genetically conditioned by a recessive allele of the $b$ gene (bolting) [8]. The dominant allele $B$ makes vernalization unnecessary for flowering. The detailed molecular organization of the $B$ locus was elucidated, and a DNA marker discriminating $b$ from $B$ was reported [8]. If the $B$ gene does not interfere with genes governing callus formation and plant regeneration in $\mathrm{NK}-219 \mathrm{~mm}-\mathrm{O}$, it may be possible to breed an annual sugar beet that is suitable for molecular genetic analysis.

First, we wished to determine the inheritance of callus formation and plant regeneration of NK-219mm-O. The parental sugar beet lines were five inbred lines, NK219mm-O, NK-195mm-O, NK-235mm-O, NK-239mm-O, and NK-294mm-O, all of which were developed by HARC for hybrid breeding using cytoplasmic male sterility (CMS), which is genetically conditioned by male sterilityinducing cytoplasm (S) and a recessive allele of $r f$ (restorer of fertility). A CMS line is produced by repeated backcrossing (more than four times) of a CMS line with a pollen parental line with the rfrf genotype but with normal fertile cytoplasm $(\mathrm{N})$ to secure pollen production. This pollen parental line is called a maintainer or an O-type in sugar beet terminology. As such, a CMS line and its cognate Otype are near-identical in nuclear genotypes but differ in their cytoplasms. CMS and O-type lines are discriminated by suffixes '-CMS' and '-O', respectively. A shared number in the prefix (e.g. '219') indicates that the two lines have nearly identical nuclear genotypes.

The five parental lines were crossed to obtain ten $F_{1}$ populations, NK-195mm-CMS x NK-219mm-O, NK195mm-CMS $x$ NK-235mm-O, NK-195mm-CMS x NK-239mm-O, NK-195mm-CMS x NK-294mm-O, NK-219mm-CMS x NK-235mm-O, NK-219mm-CMS x NK-294mm-O, NK-219mm-CMS x NK-239mm-O, NK$235 \mathrm{~mm}$-CMS $\mathrm{x}$ NK-239mm-O, NK-235mm-CMS $\mathrm{x}$ NK-294mm-O, and NK-239mm-CMS x NK-294mm-O (in this report, crosses are denoted as seed parent $\mathrm{x}$ pollen parent). We investigated the frequency of callus formation in these $F_{1}$ populations and five parental lines. As detailed in $[3,4]$, surface-sterilized seeds were sown on solid medium in vitro, and plantlets were grown. We removed two leaves from each plantlet, cut each of them into six pieces, and placed the explants on callus-inducing medium. We defined callus as apparently dedifferentiated cell clumps that were friable, white or pale yellow in color, and proliferated after detachment from the explants. The presence or absence of callus was monitored for twelve weeks. Of the two leaves, the better value was considered as the plant's phenotype for callus formation. The ratio of the number of callus-forming explants to the total number of explants was calculated for each $\mathrm{F}_{1}$ or parental line, from which fourteen to twenty plantlets were examined. A second replicate (another two leaves from each plantlets) was examined similarly (see data set in Additional file 1). We summarize these data in Table 1 and Additional file 2: Figure S1. The frequencies of callus formation for five $F_{1}$ populations involving NK-219mm-O or NK-219mm-CMS were high $(>0.92)$, a result that is comparable to that of NK-219mm-O, with the exception of NK-219mm-CMS $x$ NK-294mm-O that had a lower frequency (0.754). None of the other $F_{1}$ populations nor the parental lines exceeded a frequency of callus formation $>0.9$ except NK239mm-CMS x NK-294mm-O (0.927). The results of our quantitative genetic analyses suggested it unlikely that a complex genetic interaction is involved in callus formation (Additional file 3: Tables S1 and S2, and Additional file 2: Figure S2). Taken together, these results indicate that efficient callus formation of NK-219mm-O is a heritable character. Note that the general combining ability (GCA) and specific combining ability (SCA) are significant (Additional file 3: Table S3), suggesting that the $F_{1}$ does not always express callus formation as efficiently as NK$219 \mathrm{~mm}-\mathrm{O}$ (e.g. NK-219mm-CMS x NK-294mm-O). Heritability in a broad sense and a narrow sense is high (Additional file 3: Table S4).

Next, we examined whether the obtained calli have the potential to differentiate into shoots. The calli of the $F_{1}$ populations and the parental lines that were obtained in the dedifferentiation analysis were placed onto regeneration medium (see [3]) and grown for four weeks. Because there were no NK-239mm-O explants that generated callus (Table 1), this line was excluded from the regeneration analysis. Four weeks after transfer to the regeneration medium, calli had one of five of the following morphologies (see also Fig. 1): Type-A, many somatic embryo-like objects or adventitious shoots were evident; Type-B, very few somatic embryo-like objects or adventitious shoots were seen; Type- $C$, green objects were present that were distinct from dedifferentiated cells, somatic embryo-like objects and adventitious shoots; Type-D, calli became green but were morphologically unchanged; and Type-E, no change in color or morphology. Plantlets were obtained from somatic embryo-like objects and adventitious shoots of Types-A and $\mathrm{B}$ but not from the Type-C objects nor from TypesD or E calli. Sections of the green objects recovered 
Table 1 Half-diallel table of in vitro-culture response in $\mathrm{F}_{1}$ populations and their parental lines ${ }^{\mathrm{a}}$

\begin{tabular}{|c|c|c|c|c|c|}
\hline & NK-195mm & NK-219mm & $\mathrm{NK}-235 \mathrm{~mm}$ & NK-239mm & NK-294mm \\
\hline \multicolumn{6}{|c|}{ a) Frequencies of callus formation (\%; mean $\pm S D$ ) } \\
\hline NK-195mm & $0.08 \pm 0.15$ & & & & \\
\hline NK-219mm & $0.93 \pm 0.13$ & $0.99 \pm 0.04$ & & & \\
\hline $\mathrm{NK}-235 \mathrm{~mm}$ & $0.38 \pm 0.40$ & $0.97 \pm 0.12$ & $0.51 \pm 0.49$ & & \\
\hline NK-239mm & $0.28 \pm 0.32$ & $0.75 \pm 0.27$ & $0.50 \pm 0.43$ & $0.00 \pm 0.00$ & \\
\hline $\mathrm{NK}-294 \mathrm{~mm}$ & $0.35 \pm 0.32$ & $0.94 \pm 0.11$ & $0.79 \pm 0.17$ & $0.93 \pm 0.12$ & $0.40 \pm 0.24$ \\
\hline \multicolumn{6}{|c|}{ b) Plant regeneration scores (mean $\pm \mathrm{SD}$ ) } \\
\hline NK-195mm & $125.00 \pm 75.00$ & & & & \\
\hline $\mathrm{NK}-219 \mathrm{~mm}$ & $139.39 \pm 57.63$ & $174.47 \pm 26.27$ & & & \\
\hline $\mathrm{NK}-235 \mathrm{~mm}$ & $11.91 \pm 15.96$ & $100.86 \pm 34.78$ & $1.52 \pm 5.03$ & & \\
\hline NK-239mm & $91.13 \pm 76.57$ & $41.14 \pm 44.30$ & $47.37 \pm 45.90$ & - & \\
\hline $\mathrm{NK}-294 \mathrm{~mm}$ & $52.74 \pm 62.84$ & $113.60 \pm 34.91$ & $7.30 \pm 12.16$ & $15.52 \pm 13.00$ & $6.31 \pm 12.08$ \\
\hline
\end{tabular}

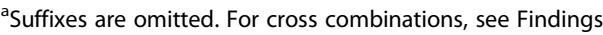

from Type-C callus were observed by light microscopy and had irregularly arranged xylem elements; whereas, normal xylem structures were present in the adventitious shoots formed on Type-A callus (see photographs $\mathrm{f}$ and $\mathrm{g}$ in Fig. 1). Therefore, Type-C callus is distinct from Types-A and B in terms of its regeneration potential. The phenotypes of calli from each genotype were characterized by calculating the percentages of each of the five phenotypes represented (Fig. 2). Phenotypes of $F_{1}$ plants appeared to be affected by their parental genotypes. For example, the NK-219mm-O genotype tended to have more Type-A or Type-B calli, whereas the NK$294 \mathrm{~mm}-\mathrm{O}$ genotype tended to have more Type-E calli.

To quantify the regeneration potential, we gave phenotypic values: 2 for Type-A calli, 1 for Type-B, and 0 for Types- $C, D$, and $E$. Using these phenotypic values, a plant's regeneration score was calculated by the following equation:

$$
\text { Regenerationscore }=\frac{2 \times N_{A}+1 \times N_{B}}{N_{\text {Total }}} \times 100
$$

in which $\mathrm{N}_{\mathrm{A}}$ is number of callus clumps exhibiting the Type-A phenotype, $N_{B}$ is number of callus clumps exhibiting the Type- $\mathrm{B}$ phenotype, and $\mathrm{N}_{\text {Total }}$ is total number of callus clumps placed on the regeneration medium. In this equation, the maximum value for the regeneration score is 200. In Table 1, the mean values of the plants' regeneration scores in the $F_{1}$ populations and parental lines are shown. The regeneration scores exceeded 100 in NK-195mm-O, NK-219mm-O, and $F_{1}$ populations involving NK-219mm-O or NK-219mm-CMS with the exception of NK-219mm-CMS x NK-294mm-O (Table 1). Our quantitative genetic analyses suggested it unlikely that a complex genetic interaction is involved in the regeneration potential (Additional file 3: Tables S5 and S6, and Additional file 2: Figure S3). Because of the significance
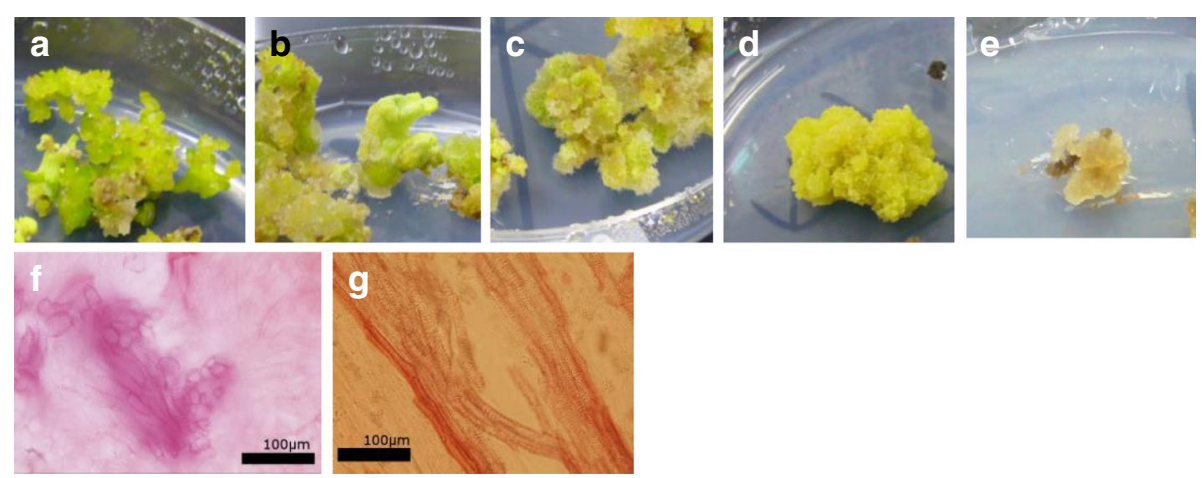
Photographs $\mathbf{f}$ and $\mathbf{g}$ are sections of green objects found on Type-C callus and adventitious shoots on Type-A callus, respectively. Objects were embedded in $5 \%$ agar and sectioned into 50- $\mu$ m slices using a Microslicer DTK-1000 (Dosaka EM, Kyoto, Japan). Before making microscopic observations (Olympus BX50 equipped with Olympus DP70, Olympus, Tokyo, Japan), the sections were stained with safranin 


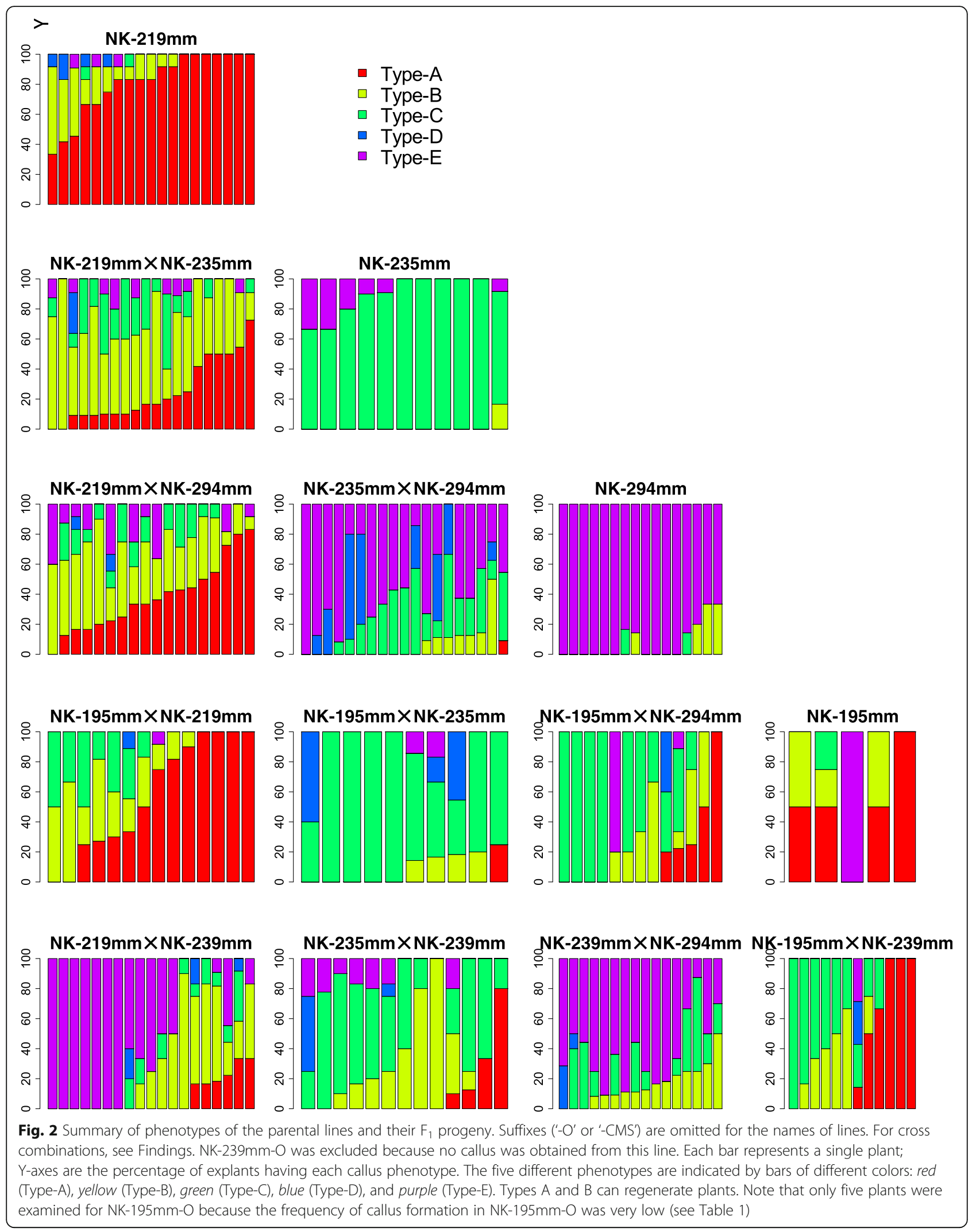


for both the GCA and the SCA (Additional file 3: Table S7), efficient plant regeneration may not always be expressed in the $F_{1}$, as exemplified in NK-219mm-CMS $x$ NK-294mm-O. Altogether, our results indicate that efficient plant regeneration of $\mathrm{NK}-219 \mathrm{~mm}-\mathrm{O}$ is a heritable character. A correlation between the frequencies of callus formation and the plant regeneration scores was unlikely considering that the correlation coefficient between these two values was insignificant $(r=0.278)$. Nevertheless, since both callus formation and plant regeneration of NK$219 \mathrm{~mm}-\mathrm{O}$ were highly heritability in a broad and narrow sense (Additional file 3: Tables S4 and S8), it is possible to select a genotype that is superior in terms of tissue culture response.

We next examined the effect of the $B$ gene on in vitro culture. TA-33BB-CMS and TA-33BB-O are annual sugar beet lines developed at the HARC. Pin et al. [8] isolated the $B$ gene through map-based cloning and showed that it encodes a pseudo-response regulator protein named BvBTC1. We determined part of the nucleotide sequence of $B v B T C 1$ cDNA from TA-33BB-O, and the data indicated that the annual habitat of TA-33BB-O is conditioned by the dominant $B$ allele (Additional file 2: Figure $\mathrm{S} 4$ ).

We also examined callus formation and the regeneration potential of TA-33BB-O, TA-33BB-CMS, NK-219mm-O $x$ TA-33BB-O, and TA-33BB-CMS x NK-219mm-O. Frequencies of callus formation were 0.1-0.11 for TA-33BB-O and TA-33BB-CMS, and 0.99-1.0 for the two $F_{1}$ populations (Additional file 2: Figure S5 and Additional file 3: Table S9). Plant regeneration scores were 77-78 for TA33BB-O and TA-33BB-CMS, and 182-200 for the two $\mathrm{F}_{1}$ populations (Additional file 3: Table S9). We obtained regenerated plants from calli of the two $F_{1}$ populations (see Additional file 2: Figure S6). The regenerated plants flowered under 24-h day conditions without vernalization. These $F_{1}$ populations can be propagated as somatic clones by in vitro culture and used for transgenic analyses.

\section{Additional files}

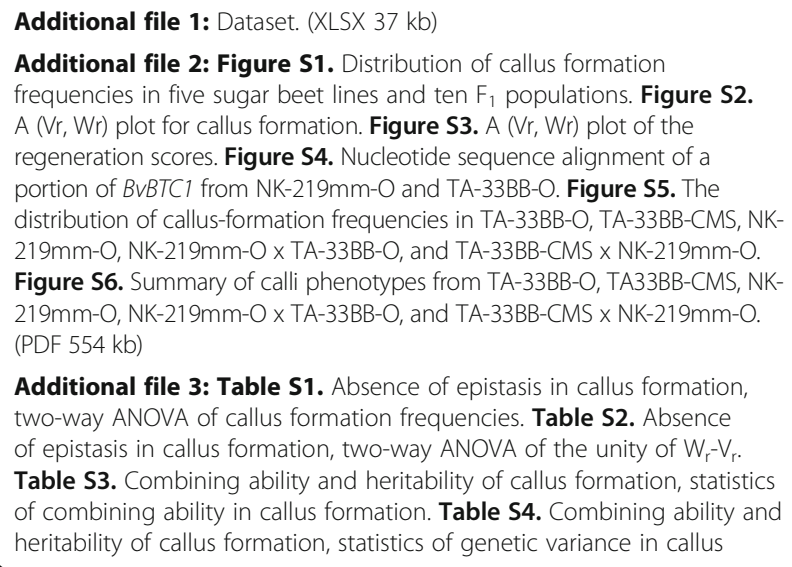

Additional file 3: Table S1. Absence of epistasis in callus formation, two-way ANOVA of callus formation frequencies. Table S2. Absence of epistasis in callus formation, two-way ANOVA of the unity of $W_{r}-V_{r}$. Table S3. Combining ability and heritability of callus formation, statistics of combining ability in callus formation. Table S4. Combining ability and heritability of callus formation, statistics of genetic variance in callus

formation. Table S5. Absence of epistasis in plant regeneration, two-way ANOVA of the regeneration score. Table S6. Absence of epistasis in plant regeneration, two-way ANOVA of the unity of $W_{r}-V_{r}$. Table S7. Combining ability and heritability of plant regeneration, statistics of combining ability in plant regeneration. Table S8. Combining ability and heritability of plant regeneration, statistics of combining ability in plant regeneration. Table S9. In vitro performances of TA-33BB-O, TA-33BB-CMS, NK-219mm-O, and their $\mathrm{F}_{1}$ populations. (PDF $110 \mathrm{~kb}$ )

\section{Abbreviations}

$B(b)$ : Bolting; CMS: Cytoplasmic male sterility; HARC: Hokkaido Agricultural Research Center; N: Normal fertile cytoplasm; rf: Restorer of fertility; S: Male sterility-inducing cytoplasm

\section{Acknowledgements}

The authors would like to thank Chisato Osawa for technical support. TA is a recipient of JSPS Research Fellowship for Young Scientists.

\section{Funding}

This work was supported in part by JSPS KAKENHI Grant Numbers 22580001 and 25292001 (TK), and Grant-in-Aid for JSPS Fellows 16 J01146 (TA).

\section{Availability of data and materials}

Because plant materials used in this study are breeding materials, they are not freely available. Those who belong to a public sector organization may use these materials by negotiating a contract with HARC.

\section{Authors' contributions}

$K T, H T$, and TK designed this study. HK, YK and KT developed plant materials. HK and TA performed in vitro culture and genotyping. HK, KT, TA and TK analyzed the data. HK, TA and TK wrote the manuscript. KT and HT performed critical comments on the draft. All authors read and approved the final version of the manuscript.

\section{Competing interests}

The authors declare that they have no competing interests.

\section{Consent for publication}

Not applicable.

\section{Ethics approval and consent to participate}

Not applicable.

\section{Author details}

${ }^{1}$ Research Faculty of Agriculture, Hokkaido University, Sapporo 060-8589, Japan. ${ }^{2}$ Hokkaido Agricultural Research Center, National Agriculture and Food Research Organization, Memuro 082-0081, Japan. ${ }^{3}$ Central Agricultural Experimental Station, Agriculture Research Department, Hokkaido Research Organization, Takikawa 073-0013, Japan.

Received: 25 June 2016 Accepted: 14 October 2016

Published online: 15 November 2016

References

1. Wang K. Agrobacterium protocols. 3rd ed. New York: Springer Science + Buisiness Media; 2015.

2. Tomita K-i, Hiura S, Tamagake H. Evaluation of the potential for somatic embryogenesis in sugar beet (Beta vulgaris L.) breeding lines and improvement of regeneration efficiency. Plant Biotechnol. 2013;30:479-87.

3. Kagami H, Kurata M, Matsuhira H, Taguchi K, Mikami T, Tamagake H, Kubo T. Chapter 27 Sugar Beet (Beta vulgaris L.). In: Wang K, editor. Agrobacterium Protocols. New York: Springer Science + Business Media; 2015. p. 335-47.

4. Kubo T. Sugar beet transformation. http://www.agr.hokudai.ac.jp/ikushu/ gelab/Sugar_beet_transformation.htm. Accessed 4 Nov 2016.

5. Matsuhira H, Kagami H, Kurata M, Kitazaki K, Matsunaga M, Hamaguchi $Y$, et al. Unusual and typical features of a novel restorer-of-fertility gene of sugar beet (Beta vulgaris L.). Genetics. 2012;192:1347-58.

6. Matsuhira H, Tamura K, Tamagake H, Sato Y, Anzai H, Yoshida M. High production of plant type levan in sugar beet transformed with timothy (Phleum pratense) 6-SFT genes. J Biotechnol. 2014;192:215-22. 
7. Kitazaki K, Arakawa T, Matsunaga M, Yui-Kurino R, Matsuhira H, Mikami T, et al. Post-translational mechanisms are associated with fertility restoration of cytoplasmic male sterility in sugar beet (Beta vulgaris). Plant J. 2015;83:290-9.

8. Pin PA, Zhang W, Vogt SH, Dally N, Büttner B, Schulze-Buxloh G, et al. The role of a pseudo-response regulator gene in life cycle adaptation and domestication of beet. Curr Biol. 2012;22:1095-101.

Submit your next manuscript to BioMed Central and we will help you at every step:

- We accept pre-submission inquiries

- Our selector tool helps you to find the most relevant journal

- We provide round the clock customer support

- Convenient online submission

- Thorough peer review

- Inclusion in PubMed and all major indexing services

- Maximum visibility for your research

Submit your manuscript at www.biomedcentral.com/submit 